\title{
An Extended Zel'dovich Model for the Halo Mass Function
}

\section{Seunghwan Lim \& Jounghun Lee}

Astronomy Program, FPRD, Department of Physics and Astronomy, Seoul National University, Seoul 151-747, Korea

E-mail: shlim@astro.snu.ac.kr, jounghun@astro.snu.ac.kr

\begin{abstract}
A new way to construct a fitting formula for the halo mass function is presented. Our formula is expressed as a solution to the modified Jedamzik matrix equation that automatically satisfies the normalization constraint. The characteristic parameters expressed in terms of the linear shear eigenvalues are empirically determined by fitting the analytic formula to the numerical results from the high-resolution N-body simulation and found to be independent of scale, redshift and background cosmology. Our fitting formula with the best-fit parameters is shown to work excellently in the wide mass-range at various redshifts: The ratio of the analytic formula to the N-body results departs from unity by up to $10 \%$ and $5 \%$ over $10^{11} \leq M /\left(h^{-1} M_{\odot}\right) \leq 5 \times 10^{15}$ at $z=0,0.5$ and 1 for the FoF-halo and SO-halo cases, respectively.
\end{abstract}

Keywords: Cosmology, Halo Mass Function 


\section{Contents}

1 Introduction 1

2 Analytic framework: a review 2

3 Extension of the Zel'dovich model 4

4 Numerical tests $\quad 6$

4.1 Determination of the barrier heights $\quad 6$

4.2 Direct comparison with the N-body results 10

$\begin{array}{lll}4.3 & \text { Dependence on the filter shape } & 12\end{array}$

5 Discussion $\quad 14$

\section{Introduction}

The era of precision cosmology that reigned supreme in the last century has waned, rendering its glory to the advent of a new era of accurate cosmology [1]. While the goal of the previous era was the precise measurements of a model-dependent finite set of the cosmological parameters, the accurate cosmology aims at addressing more fundamental issues such as what the origin of cosmic inflation is, what the limitation of the general relativity is, what drove the cosmic acceleration and etc, which requires to explore almost infinite parameter space spanned by all possible cosmological and physical scenarios.

In precision era, resorting solely to the high-resolution simulations suffices to make precise theoretical predictions for the determination of the cosmological parameters. However, in accuracy era, given that it is extremely expensive and inefficient to sweep through the infinite parameter space using the simulations alone, developing robust analytical guidelines is highly desired to complement the numerical experiments. The mass function of bound halos has been spotlighted as one of those few cosmological probes which allow an analytical approach.

It was ref. [2, hereafter PS] who developed for the first time an analytic formalism for the halo mass function under several simplified assumptions on the halo formation process. Later, ref. [3] introduced the excursion set theory to analytically derive the mass function from physical principles and found that the excursion set theory yields the same PS mass function for the special case of the sharp- $k$ space filter [see also 4]. In the light of these landmark works, rapid progress has been made to improve the original excursion set formalism in two different directions. One direction was to refine the analytical prescriptions by incorporating into the excursion set theory more realistic aspects of the halo formation process such as the occurrence of the clouds-inclouds, spatial correlations among the proto-halos, tidal effect from the surrounding matter distribution, diffusive nature of the density threshold, and etc [e.g., 5-19]. 
The other direction focused on finding a more accurate analytic formula for the halo mass function by modifying the functional form of the excursion set mass function [20-27]. The modified functional forms are characterized by free parameters whose best-fit values have to be empirically determined by fitting the formulae to the highresolution N-body results. Although the recent analytic formulae have been found to agree excellently with the N-body results, the best-fit values of their characteristic parameters turned out to likely vary with the mass scale, redshift and background cosmology [e.g., 26, 28].

In this paper, we present a new fitting formula for the halo mass function which is characterized by the free parameters independent of scale, redshift and background cosmology. The Jedamzik formalism [5] is adopted as our statistical framework within which the halo mass function is automatically normalized. Throughout this paper, we assume a flat $\Lambda$ CDM cosmology with the WMAP7 parameters [30] (unless stated otherwise) and use the CAMB code [31] for the evaluation of the $\Lambda$ CDM linear power spectrum.

\section{Analytic framework: a review}

Most of the previous formulae for the halo mass functions were constructed in the the following analytic framework [20-27]:

$$
\frac{d N(M, z)}{d \ln M}=\frac{\bar{\rho}}{M} \frac{d \ln \sigma^{-1}}{d \ln M} f[\sigma(M, z)] .
$$

Here $d N(M, z) / d \ln M$ is the differential number density of the bound halos in a logarithmic mass interval of $[\ln M, \ln M+d \ln M]$ at redshift $z$ per unit volume, $\sigma(M, z) \equiv b(z) \sigma(M, 0)$ is the rms fluctuation of the linear density field smoothed on the mass scale $M$ at redshift $z$ where $b(z)$ is the linear growth factor satisfying the condition of $b(0)=1, \bar{\rho}$ is the mean mass density of the Universe, and $f(\sigma)$, called the multiplicity function, represents a differential volume fraction occupied by those regions which satisfy a prescribed collapse condition for the halo formation. In jargon of the excursion set theory, a random walk proceeds as the time-like variable $\sigma$ increases until it hits a specified collapse barrier. The number of those random walks which first cross a given collapse barrier on scale of $\sigma(M, z)$ is proportional to the number of the bound halos of mass $M$ formed at $z[3]$.

The functional form of $f(\sigma)$ that is the key quantity in equation (2.1) depends on the correlations among random walks as well as on the shape of the collapse barrier. The latter is determined by the underlying dynamics while the former is related to the shape of the filter used to smooth the linear density field. As mentioned in section 1, ref. [3] showed that the original PS mass function can be derived from the excursion set formalism under the assumptions that the random walks are Markovian (i.e., uncorrelated) corresponding to the sharp- $k$ space filter and that the collapse barrier has a flat shape, $\delta=\delta_{s c}$, corresponding to the spherical dynamics. The PS multiplicity 
function derived from the excursion set theory is written as

$$
f_{\mathrm{PS}}[\sigma(M, z)]=\sqrt{\frac{2}{\pi}} \frac{\delta_{s c}}{\sigma(M, z)} \exp \left[-\frac{\delta_{s c}^{2}}{2 \sigma^{2}(M, z)}\right]
$$

where the height of the spherical collapse barrier $\delta_{s c} \simeq 1.686$ depends only very weakly on the background cosmology [32].

As also mentioned in section 1 , much effort has been made to find a better fitting formula by phenomenologically modifying the above PS multiplicity function. For instance, ref. [25] modified the PS multiplicity function into

$$
f_{\text {tinker }}(\sigma)=A\left[\left(\frac{\sigma}{b}\right)^{-a}+1\right] \exp \left(-\frac{c}{\sigma^{2}}\right)
$$

where the four coefficients, $A, a, b$ and $c$, are free parameters whose best-fit values were determined empirically by comparing equation (2.3) with the N-body results. Their formula were found to agree with the high-resolution N-body results with accuracy up to maximum $10 \%$ error for the case that a bound halo is identified by the friends-offriends (FoF) algorithm [33].

For the case that a bound halo is identified by the spherical overdensity algorithm [34], the following formula for the multiplicity function provided by ref. [27] has been found to work better [see also 23, 24]:

$$
f_{\text {pillepich }}(\sigma)=\left[D+B\left(\frac{1}{\sigma}\right)^{A}\right] \exp \left(-\frac{C}{\sigma^{2}}\right) .
$$

The best-fit values of the four coefficients $A, B, C$ and $D$ were determined by comparing equation (2.4) to the results from the high-resolution N-body simulations for a $\Lambda \mathrm{CDM}$ cosmology with the WMAP5 parameters [35]. Their formula was demonstrated to reach the accuracy level up to maximum $5 \%$ error over a wide mass range of $2.4 \times 10^{10} \leq$ $M /\left(h^{-1} M_{\odot}\right) \leq 10^{15}$. For the other fitting formulae formulated within the framework of equation (2.1), see Table 7 in ref. [27].

It is worth noting that the multiplicity function in equation (2.1) does not automatically satisfy the normalization constraint of $\int_{0}^{\infty} d \sigma f(\sigma)=1$ (under the assumption that all initial regions would eventually collapse to form bound halos) but its overall amplitude had to be treated as an additional fitting parameter. For instance, the parameter $A$ in equation (2.3) and $D$ in equation (2.4) had to be introduced to satisfy the normalization constraint.

It has been conventionally thought that the normalization of the halo mass function is related to the issue of taking into account the occurrence of the clouds-in-clouds (underdense regions embedded in larger overdense regions) and that for the special case of Markovian random walks with flat spherical collapse barrier the overall normalization factor of 2 introduced by PS can be justified by the excursion set theory [3-5] as a solution to the clouds-in-clouds problem. Very recently, however, ref. [17] argued that the normalization of the halo mass function in fact reflects the limitation 
of the excursion set approach itself having strong dependence on the correlations of random walks.

Given the downside of equation (2.1) that it requires an additional free parameter to satisfy the normalization of the halo mass function, here we instead consider the Jedamzik equation [5] as our analytic framework which automatically yields the normalized mass function:

$$
F(M)=\int_{M}^{\infty} d M^{\prime} \frac{M^{\prime}}{\bar{\rho}} \frac{d N}{d M^{\prime}} P\left(M, M^{\prime} ; M^{\prime} \text { being isolated }\right)
$$

where $F(M)$ is the cumulative volume fraction occupied by those random walks which exceed the collapse barrier on the mass scale $M$, while $P\left(M, M^{\prime} ; M^{\prime}\right.$ bing isolated) is the conditional probability that a random walk exceeds the collapse barrier on the mass scale $M$ provided that it for the first time just touched the collapse barrier on some larger mass scale $M^{\prime}$. That is, the isolated $M^{\prime}$ means that the random walk has never touched (nor exceeded) the collapse barrier on mass scales larger than $M^{\prime}$.

For the special case of the sharp- $k$ space filter (Markovian random walks), the conditional probability $P\left(M, M^{\prime} ; M^{\prime}\right.$ bing isolated $)$ can be simply given as the conditional probability $P\left(M, M^{\prime}\right)$ that a random walk exceeds the collapse barrier on the mass scale $M$ provided it just touched the collapse barrier on some larger mass scale $M^{\prime}$ [5]. For non-Markovian case (corresponding to the cases of the top-hat or Gaussian filters), however, we have inequality $P\left(M, M^{\prime} ; M^{\prime}\right.$ bing isolated $) \neq P\left(M, M^{\prime}\right)$, and thus it is expected that $P\left(M, M^{\prime} ; M^{\prime}\right.$ bing isolated) would have much more complicated expression. Nontheless, in the current work, we use $P\left(M, M^{\prime}\right)$ for the conditional probability in equation (2.5), since our goal here is not to estalish a physical model but just to find a new fitting formula for the halo mass function which is otherwise devoid of physical content.

\section{Extension of the Zel'dovich model}

The original Jedamzik formalism employed the spherical collapse dynamics for which the collapse barrier is flat (i.e., scale-independent), expressed in terms of the linear density contrast, just as in the original PS formalism. It has long been realized, however, that the condition for a given Lagrangian region to form a bound halo should depend not only on its initial spherically averaged overdensity but also on the tidal shears from the surrounding matter distribution [e.g., 7-9, 11, 20]. The tidal shears have an effect of disturbing the gravitational collapse and deviating the collapse process from spherical symmetry. To take into account the tidal shear effect, the gravitational collapse process should be described by more realistic ellipsoidal dynamics rather than by the simplified spherical dynamics. Unlike the spherical case, however, there is no unique collapse condition for the case of the ellipsoidal collapse process [36]. Among several different ellipsoidal dynamics that were used in the literature, the Zel'dovich approximation [29] is one of those models which can be relatively easily implemented into the mass function formalism since its ellipsoidal collapse condition is simply given 
in terms of the three eigenvalues, $\lambda_{1}, \lambda_{2}, \lambda_{3}$ (in a decreasing order, $\lambda_{1} \geq \lambda_{2} \geq \lambda_{3}$ ) of the initial shear tensors $\left(T_{i j}\right)$.

In several literatures, the Zel'dovich approximation has already been used as an underlying dynamics for the halo mass function. For example, refs. [8, 9] assumed that the gravitational collapse occurs when the largest shear eigenvalue, $\lambda_{1}$, reaches some critical value, $\lambda_{1 c}$, which corresponds to the collapse along the first principal axes of the initial shear tensors in the Zel'dovich approximation. Ref. [20] derived the halo mass function with ellipsoidal collapse barrier of $\lambda_{3}=\lambda_{3 c}$ that corresponds to the third principal axis collapse [see also 7] according to the dynamical guideline of the Zel'dovich approximation.

Those preivous works which use the Zel'dovich approximation as a dynamical guideline assume that the gravitational collapse occurs when the shear eigenvalues are all positive. Unfortunately, however, ref. [37] have shown that one of the initial shear eigenvalues at a proto-halo region has different sign from the other two, which basically invalidates this fundamental assumption. Henceforth, those previous models based on the Zel'dovich approximation are not physical ones but only mechanistic prescriptions to determine the fitting formula for the halo mass function. Apart from the invalid collapse condition, the previous works based on the Zel'dovich model also suffered from the normalization problem which is attributed to the fact that even for the case of the sharp- $k$ space filter $P\left(M, M^{\prime} ; M^{\prime}\right.$ bing isolated $) \neq P\left(M, M^{\prime}\right)$ if the collapse conditions are expressed in terms of the shear eigenvalues. Very recently, ref. [38] have suggested that the normalization of the halo mass function in the Zel'dovich model can be satisfied if the Jedamzik formalism is used as a statistical framework.

Adopting a similar statistical strategy to that of ref.[38], we construct an extended Zel'dovich model (EZL) for the halo mass function for three different cases: For the one-dimensional case (1D EZL), the free parameter is given as the threshold of the smallest shear eigenvalue: $\vec{\lambda}_{c}=\left\{\lambda_{3 c}\right\}$. For the two dimensional case (2D EZL), theere are two free parameters given as the thresholds of the second to the largest and the smallest eigenvalues: $\vec{\lambda}_{c}=\left\{\lambda_{2 c}, \lambda_{3 c}\right\}$, while for the three dimensional case (3D EZL), there are three free parameters given as the thresholds of all three shear eigenvalues: $\vec{\lambda}_{c}=\left\{\lambda_{1 c}, \lambda_{2 c}, \lambda_{3 c}\right\}$. We emphasize here that the thresholds of the shear eigenvalues are not true physical collapse barriers but just free parameters that characterize the halo mass function in the mechanistic Zel'dovich model.

Expressing the free parameters in terms of the shear eigenvalues, we modify the Jedamzik framework as

$$
F\left(M, \vec{\lambda}_{c}\right)=\int_{M}^{\infty} d M^{\prime} \frac{M^{\prime}}{\bar{\rho}} \frac{d N}{d M^{\prime}} P\left(M, M^{\prime}, \vec{\lambda}_{c}\right) .
$$

Here $F\left(M, \vec{\lambda}_{c}\right)$ is the cumulative probability that the initial shear eigenvalues exceed the threshold values of $\vec{\lambda}_{c}$ on the mass scale of $M$, while $P\left(M, M^{\prime}, \vec{\lambda}_{c}\right)$ is the conditional probability that the shear eigenvalues on the mass scale of $M$ exceed the thresholds of $\vec{\lambda}_{c}$ provided that the shear eigenvalues on some larger mass scale of $M^{\prime}$ just equal the thresholds. 
In equation (3.1) the cumulative probability, $F\left(M, \vec{\lambda}_{c}\right)$, can be analytically evaluated by integrating the three-point probability density distribution, $p\left(\lambda_{1}, \lambda_{2}, \lambda_{3 c}\right)$, which was derived by [39]:

$$
F\left(M, \vec{\lambda}_{c}\right)=\int_{C} \Pi_{i=1}^{n} d^{n} \lambda_{i} p(\vec{\lambda} ; \sigma),
$$

where $n=3,2,1$ for the cases of the 3D, 2D, and 1D EZL, respectively. Here the lower-bound $C$ represents the multi-dimensional area over which the integration is performed. For the 1D EZL, it is $\left\{\lambda_{1} \geq \lambda_{2} \geq \lambda_{3} \geq \lambda_{3 c}\right\}$, while for the 2D and 3D EZL, they are $\left\{\lambda_{1} \geq \lambda_{2} \geq \lambda_{2 c}, \lambda_{1} \geq \lambda_{2} \geq \lambda_{3} \geq \lambda_{3 c}\right\}$, and $\left\{\lambda_{1} \geq \lambda_{1 c}, \lambda_{1} \geq \lambda_{2} \geq \lambda_{2 c}, \lambda_{1} \geq\right.$ $\left.\lambda_{2} \geq \lambda_{3} \geq \lambda_{3 c}\right\}$, respectively

The conditional probability, $P\left(M, M^{\prime}, \vec{\lambda}_{c}\right)$, in equation (3.1) can be also obtained under the assumption of Markovian random walks as

$$
\begin{aligned}
P\left(M, M^{\prime}, \vec{\lambda}_{c}\right) & =P\left(\vec{\lambda} \geq \vec{\lambda}_{c} \mid \vec{\lambda}^{\prime}=\vec{\lambda}_{c}\right) \\
& =\int_{C} d^{n} \lambda_{i} p\left(\vec{\lambda} \mid \vec{\lambda}^{\prime}=\lambda_{c}\right)=\frac{p\left(\vec{\lambda} \geq \vec{\lambda}_{c}, \vec{\lambda}^{\prime}=\lambda_{c}\right)}{p\left(\vec{\lambda}^{\prime}=\lambda_{c}\right)},
\end{aligned}
$$

where $\vec{\lambda}$ and $\vec{\lambda}^{\prime}$ denote the shear eigenvalues on two different mass scales $M$ and $M^{\prime}$, respectively. The six-point joint probability density distribution, $p\left(\vec{\lambda}, \vec{\lambda}^{\prime}\right)$, has been derived analytically by [40] and [41]. For a practical calculation, we discretize equation (3.1) and reexpress it as a matrix product. Then we obtain the halo mass function $d N / d \ln M$ as a column vector by converting the matrix equation.

It is worth noting that our 1D EZL formula is very similar to that of [20] in the respect that both of the approaches employed the same ellipsoidal collapse condition of $\lambda_{3}=\lambda_{3 c}$. In the former, however, the halo mass function is automatically normalized while in the latter the halo mass function had to be multiplied wrongly by a constant of 12.5 without taking into account the scale dependence of the normalization factor. It is also worth mentioning the key differences between our EZL model and that of ref [38]. The latter work used the uppler limit on the largest shear eigenvalue, $\lambda_{1} \leq \lambda_{1 c}$ to describe the formation of marginally bound superclusters. In the current EZL model for the mass function of bound halos, there is no such upper limit on the shear eigenvalue. In the next section we determine the best-fit values of the free parameters of our EZL formulae by fitting them to the numerical results from the high-resolution N-body simulations.

\section{Numerical tests}

\subsection{Determination of the barrier heights}

For the empirical determination of the free parameters of our EZL formula, one has to bear in mind that the numerical results from the N-body simulations depend on which halo identification algorithm is used. Among the several halo identification algorithms that have been suggested, it is the friends-of-friends (FoF) [33] and the spherical overdensity (SO) [34] algorithms that are most frequently used in the literatures. The 


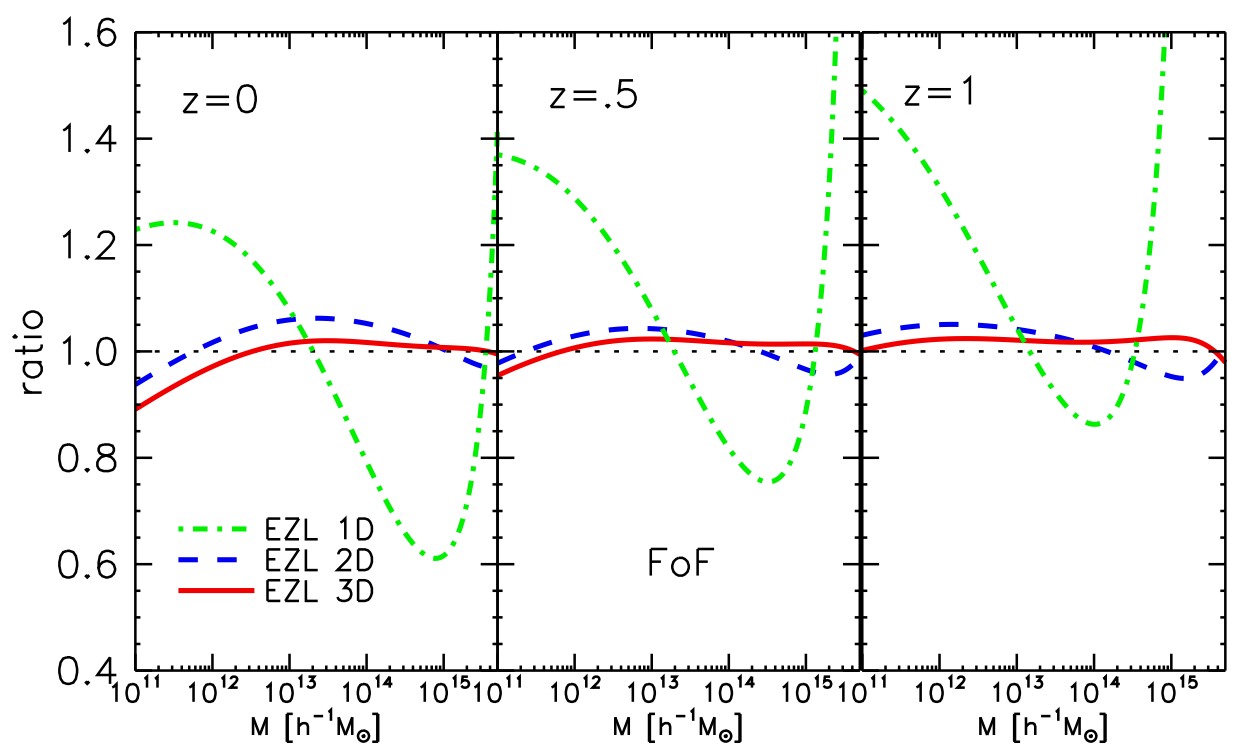

Figure 1. Ratios of the 1D, 2D and 3D EZL mass functions of the FoF halos (green dot dashed, blue dashed, and red solid lines, respectively) to the N-body results given in ref. [27] as a function of the FoF mass at three different redshifts.

former defines a bound halo as the collection of closely packed particles whose separation distances are less than some prescribed linking length, while the latter defines a bound halo as the region in which the spherically averaged density around its density peak exceeds some critical value. Given that the mass functions of FoF halos have turned out to be different from those of the SO halos [e.g., 25], we consider both of the FoF and SO cases and determine separately the free parameters of the EZL formula for each case.

For the FoF halo case, we compare our EZL mass function to the numerical results of [27, hereafter PPH10] (see eq. [2.4]). We first conduct a $\chi^{2}$-fitting of the 1D EZL formula to the PPH10 at $z=0$ by adjusting only the value of $\lambda_{3 c}$ and find the bestfit barrier height of the 1D EZL to be $\left\{\lambda_{3 c}=0.41\right\}$. The top-hat filter is used to calculate the rms density fluctuation $\sigma(M)$. Figure 1 shows the ratios of the 1D EZL mass functions with this best-fit barrier height to the numerical results of PPH10 as green dot-dashed lines over the wide mass range of $10^{11} \leq M /\left(h^{-1} M_{\odot}\right) \leq 10^{15}$ at three different redshifts ( $z=0,0.5$ and 1 in the left, middle and right panels, respectively). In each panel, the horizontal dotted line corresponds to unity. As can be seen, the agreement between the 1D EZL and the PPH10 is not so good for each case, becoming worse as $z$ increases.

We similarly conduct a $\chi^{2}$ fitting of the 2D and 3D EZL mass functions to the PPH10 by adjusting their free parameters, respectively. The best-fit parameters for 


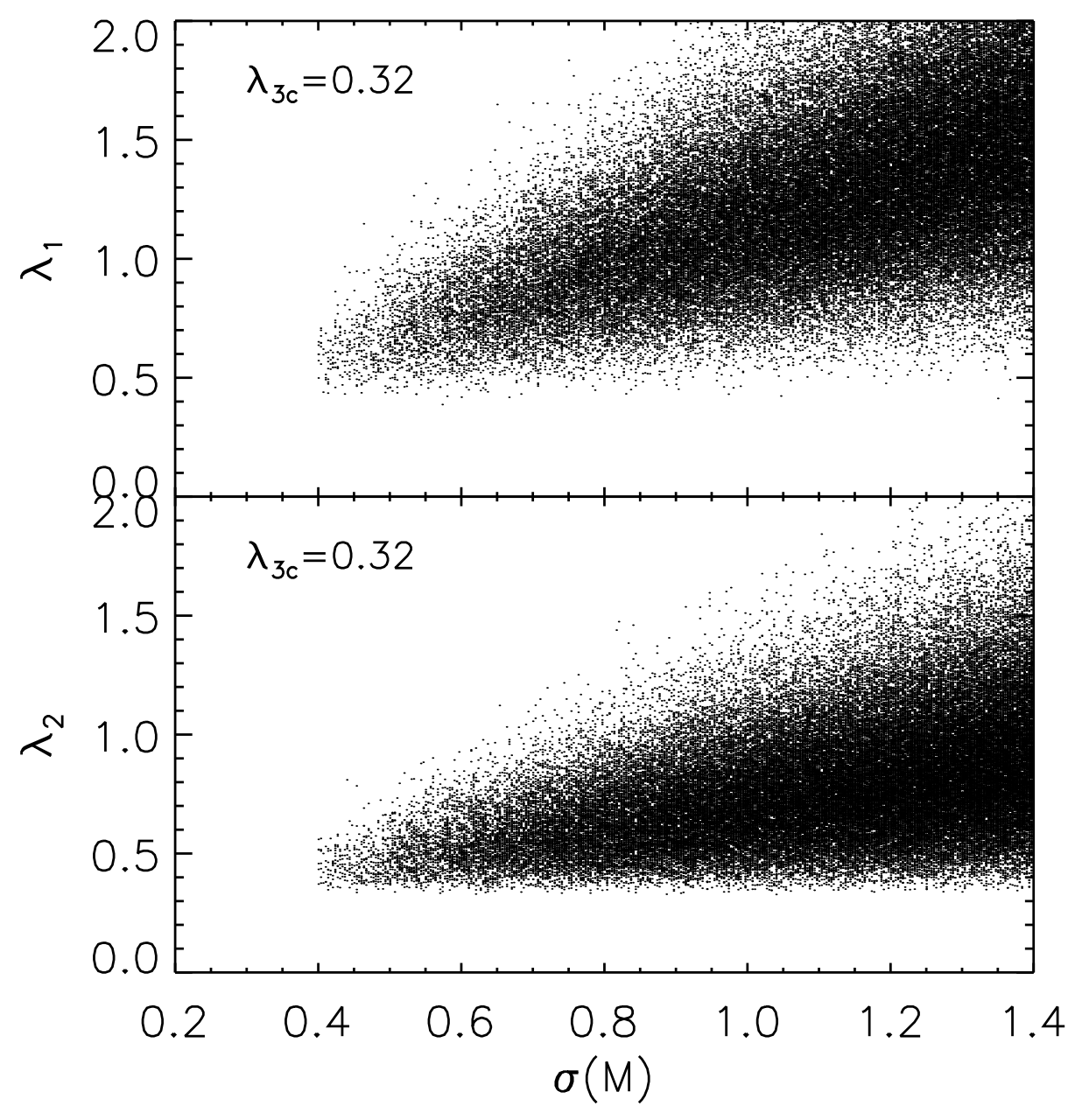

Figure 2. Scatters of the randomly generated shear eigenvalues satisfying the condition of $\lambda_{3} \geq 0.32$ in the $\sigma(M)-\lambda_{1}$ and $\sigma(M)-\lambda_{2}$ plane in the top and bottom panels, respectively.

the $2 \mathrm{D}$ and $3 \mathrm{D}$ cases are found to be $\left\{\lambda_{2 c}=0.547, \lambda_{3 c}=0.32\right\}$ and $\left\{\lambda_{1 c}=0.567, \lambda_{2 c}=\right.$ $\left.0.542, \lambda_{3 c}=0.32\right\}$, respectively. The best-fit 2D and 3D EZL mass functions are shown as blue dashed and solid red lines, respectively in Figure 1. As can be seen, the 2D and 3D EZL mass functions show much better agreements with the PPH10 than their 1D counterpart at each redshift. At all redshifts the 3D EZL mass function matches the PPH10 best in the whole mass range except for in the range of $M \leq 10^{12} h^{-1} M_{\odot}$ at $z=0$ where the 2D EZL formula works slightly better than its 3D counterpart. The ratio of the 2D (3D) EZL mass function to the PPH10 departs from unity by up to $10 \%(7 \%)$ over the whole mass range at the three redshifts.

Noting that the best-fit value of $\lambda_{2 c}$ for the 3D EZL case is very close to that for the 2D EZL case and that the 2D EZL formula works almost as well as its 3D counterpart, we investigate how the 2D EZL constraints on $\left\{\lambda_{2}, \lambda_{3}\right\}$ bias the allowed 


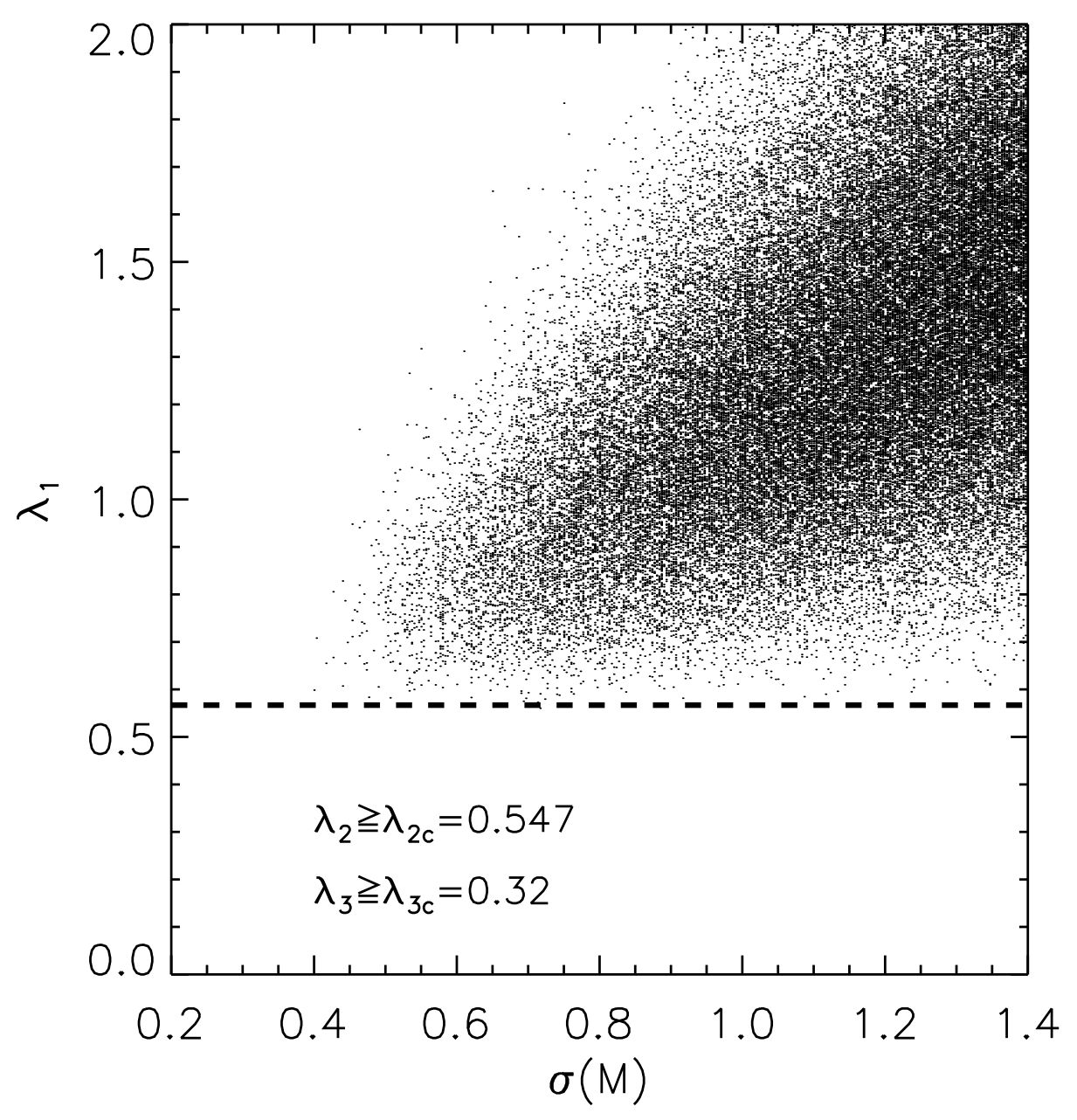

Figure 3. Scatters of the randomly generated shear eigenvalues satisfying the condition of $\left\{\lambda_{2} \geq 0.547, \lambda_{3} \geq 0.32\right\}$ in the $\sigma(M)-\lambda_{1}$ plane. The horizontal dashed line corresponds to the 3D EZL threshold of $\lambda_{1 c}=0.567$.

distribution of $\lambda_{1}$. Generating randomly a set of three shear eigenvalues on the mass scale of $M$ with the help of the Monte-Carlo algorithm developed by [10], and selecting only those random points which satisfy $\lambda_{3} \geq 0.32$, we determine the values of $\lambda_{2}$ and $\lambda_{1}$. Then we repeat the whole process on different mass scales. For the detailed description of the Monte-Carlo algorithm to generate randomly the three shear eigenvalues, see [10]. Figure 2 shows the scatter plots of the selected random points in the $\sigma(M)-\lambda_{1}$ and $\sigma(M)-\lambda_{2}$ planes in the top and bottom panels, respectively. As can be seen, there are low but non-zero probabilities on all mass scales that $\lambda_{1}$ and $\lambda_{2}$ exceed the thresholds of 0.567 and 0.542 , respectively when $\lambda_{3} \geq 0.32$ is satisfied.

We repeat the same procedure but with the two constraints of $\lambda_{2} \geq 0.547$ and $\lambda_{3} \geq 0.32$ to see how biased the values of $\lambda_{1}$ are. Figure 3 plots the result in the 


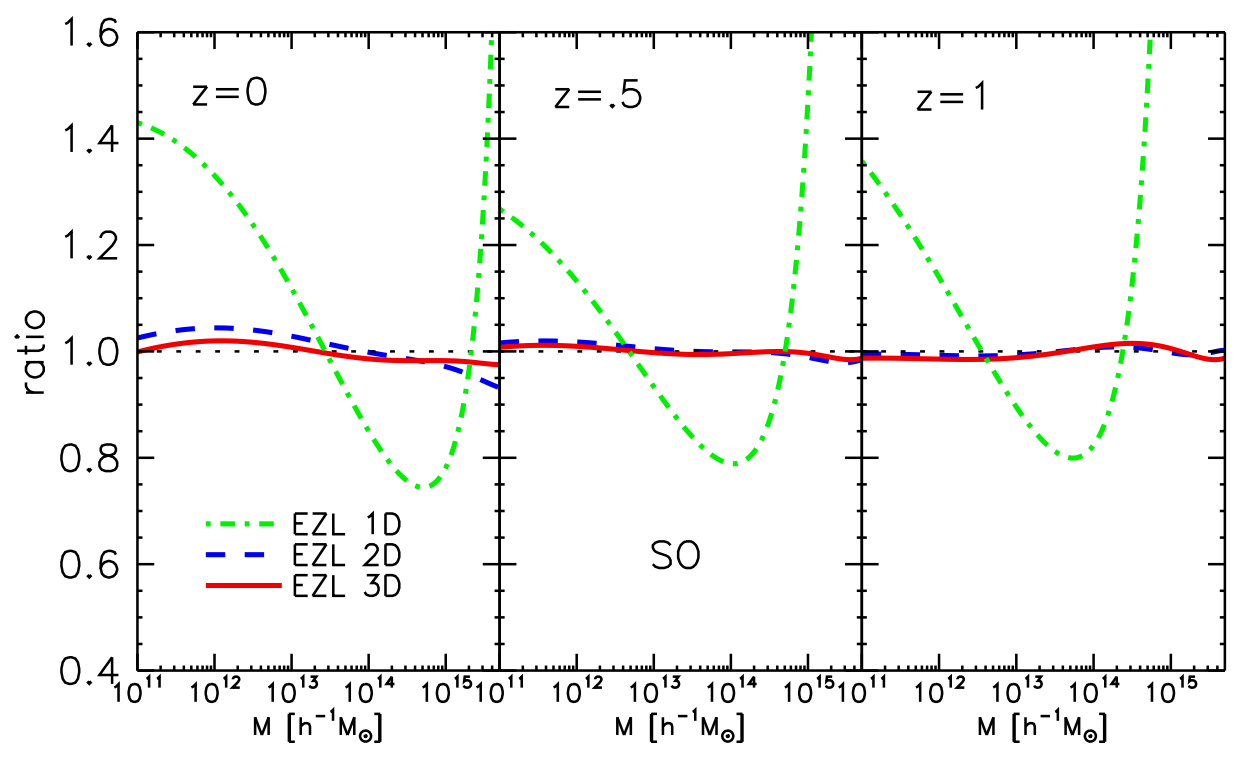

Figure 4. Same as figure 1 but using the Tinker08 formula as the numerical results to compare with the EZL mass functions of the SO halos.

$\sigma(M)-\lambda_{1}$ plane. As can be seen, the 3D EZL constraint of 0.567 is automatically satisfied by the cuts on $\lambda_{2}$ and $\lambda_{3}$, which explains why the best-fit value of $\lambda_{2 c}$ for the 3D EZL case is so close to that for the 2D EZL case as well as why the 2D EZL formula works almost as well as the 3D EZL one.

Now, let us turn to the SO halo case for which we use the numerical results of [25, hereafter, Tinker08] (see eq.[2.3]) to compare our EZL mass functions with. Repeating the same $\chi^{2}$-fitting procedure, we determine the best-fit free parameters of the $1 \mathrm{D}, 2 \mathrm{D}$, and 3D EZL formula for the SO-halo case as $\left\{\lambda_{3 c}=0.32\right\},\left\{\lambda_{2 c}=0.56, \lambda_{3 c}=0.32\right\}$ $\left\{\lambda_{1 c}=0.56, \lambda_{2 c}=0.557, \lambda_{3 c}=0.32\right\}$, respectively. Figure 4 shows the same as figure 1 but for the SO halo case. As can be seen,the 2D and 3D EZL mass functions agree excellently with the Tinker08 at all three redshifts. The 2D (3D) EZL mass function reaches the accuracy level of maximum 5\% (3\%) error at all three redshifts, which indicates that our EZL formula works well for the FoF and SO cases alike but slightly better for the latter case. It is worth emphasizing here that we use the same constant values of $\vec{\lambda}_{c}$ to plot the EZL formula at all three redshifts in figures 1-4, which implies that the multi- dimensional barrier heights of our EZL formaula, even though determined empirically at $z=0$, are redshift- independent.

\subsection{Direct comparison with the N-body results}

In this section we compare the EZL formula directly with the numerical results from two different N-body simulations. First, we use the catalog of the FoF halos identified in 


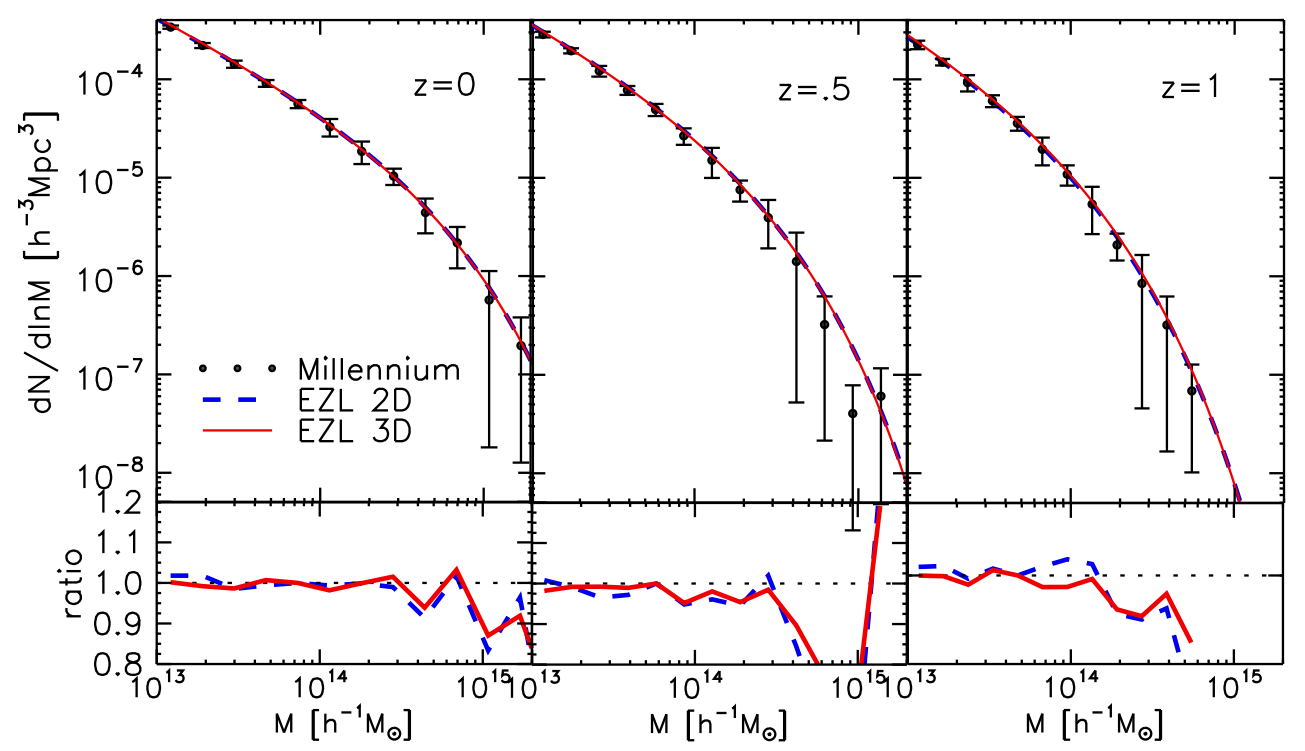

Figure 5. (Top panel): Comparison of the EZL mass functions with the numerical results from the Millennium simulations at three different redshifts. The parameters of the $2 \mathrm{D}$ and 3D EZL models are set at $\left\{\lambda_{2 c}=0.547, \lambda_{3 c}=0.32\right\}$ and $\left\{\lambda_{1 c}=0.567, \lambda_{2 c}=0.542, \lambda_{3 c}=\right.$ $0.32\}$, respectively. In each top panel the errors bars are obtained as the standard deviation scatter among eight Jack-knife resamples. (Bottom panel): Ratios of the EZL mass functions to the N-body results.

the Millennium simulations [42] for which the total number of particles $\left(N_{p}\right)$, individual particle mass, linear box size $\left(L_{p}\right)$ and input cosmological parameters were given as $N_{p}=10^{10}, m_{p}=8.6 \times 10^{8} h^{-1} M_{\odot}, L_{p}=0.5 h^{-1} \mathrm{Gpc}, \Omega_{m}=0.25, \Omega_{\Lambda}=0.75, h=$ $0.73, \sigma_{8}=0.9, n_{s}=1.0$, respectively.

Binning the logarithmic masses of the FoF halos from the Millennium halo catalog and counting their number densities of those FoFo halos belonging to each logarithmic mass-bin per unit volume, we numerically determine, $d N / d \ln M$, at $z=0,0.5,1$. The top panel of figure 5 compares the numerical mass functions of the FoF halos (dots) with Jack-knife errors from the Millennium simulations with the 2D EZL (blue dashed lines) and 3D EZL (red solid lines) mass functions respectively, while its bottom panels show the ratios of the EZL mass functions to the Millennium results. The Jack-knife errors are calculated as one standard deviation scatter among eight Jack-knife resamples [38]. For the plots of the EZL mass functions in each panel, we set the free parameters at the best-fit values empirically determined in section 4.1: $\left\{\lambda_{2 c}=0.547, \lambda_{3 c}=0.32\right\}$ and $\left\{\lambda_{1 c}=0.567, \lambda_{2 c}=0.542, \lambda_{3 c}=0.32\right\}$ for the 2D and 3D cases, respectively. As can be seen, both of the 2D and 3D EZL mass functions agree very well with the Millennium results at all redshifts.

We repeat the same calculations but using the catalog of the FoF halos identified 


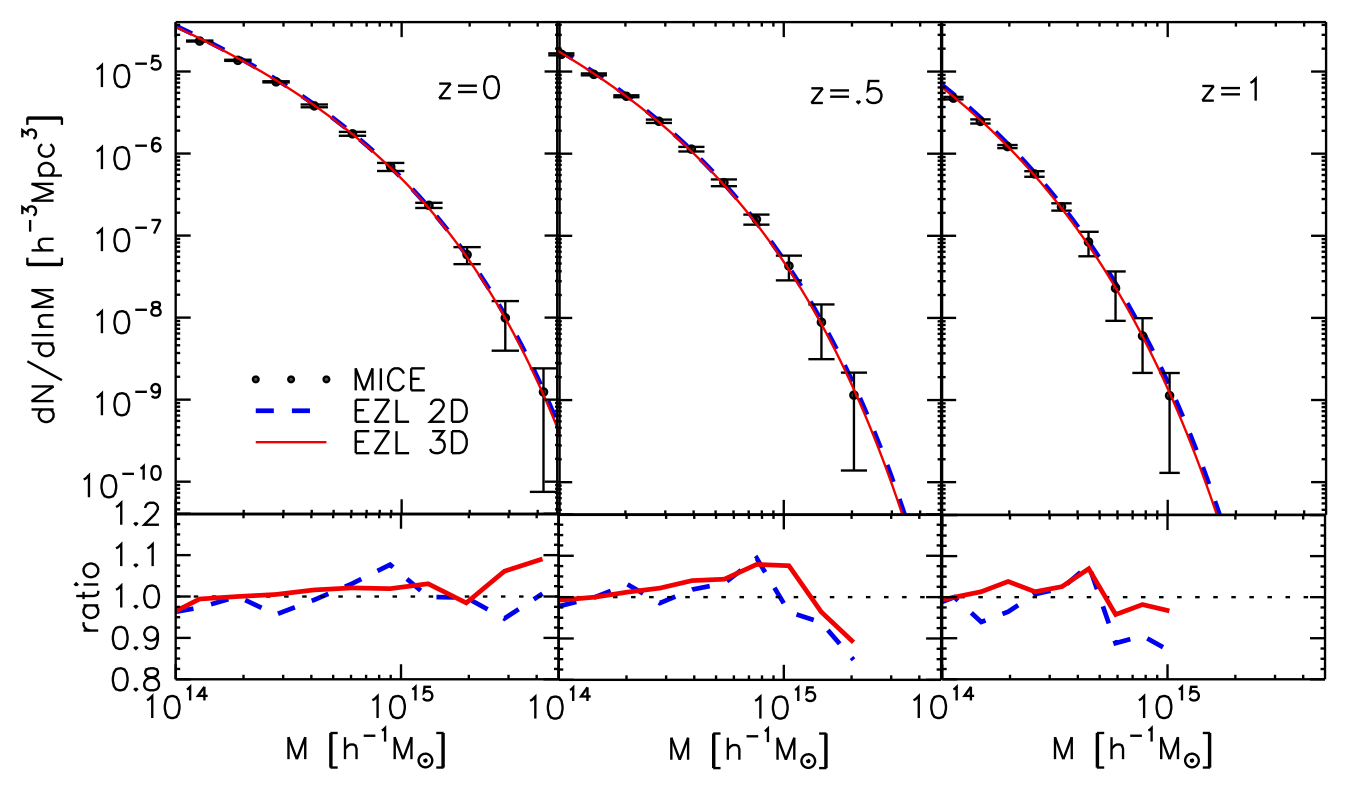

Figure 6. Same as figure 5 but with the numerical results from the MICE simulations. The same best-fit parameters that are used in figure 5 are also used for the evaluating of the 2D and 3D EZL mass functions

in the MICE simulations [26]: for which the total number of particles $\left(N_{p}\right)$, individual particle mass, linear box size $\left(L_{p}\right)$ and input cosmological parameters were given as $N_{p}=1024^{3}, m_{p}=23.42 \times 10^{10} h^{-1} M_{\odot}, L_{p}=3.072 h^{-1} \mathrm{Gpc}, \Omega_{m}=0.3, \Omega_{\Lambda}=0.7, h=$ $0.7, \sigma_{8}=0.8$, respectively. Figure 6 shows the same as figure 5 but with the numerical results from the MICE simulations. As can be seen, the EZL mass functions with the same barrier heights show excellent agreements with the MICE mass functions at all three redshifts. Given that two simulations used two different values of the power spectrum amplitude $\left(\sigma_{8}=0.9\right.$ and 0.8 for the Millennium and MICE simulation, respectively), the results shown in figures 5-6 imply that the multi-dimensional barrier height of the EZL mass function should be independent of the background cosmology.

\subsection{Dependence on the filter shape}

In the excursion set theory, the mass assignment to a bound halo depends on the shape of the filter used to calculate the rms density fluctuation $\sigma(M)$. For the results shown in figures 1-6, we have used exclusively the top-hat filter. Using a different filter is likely to alter the shape of the EZL mass function and thus accordingly the best-fit values of the multi-dimensional barrier heights. To investigate the dependence of the best-fit values of the free parameters on the filter shape, we reevaluate the 2D EZL mass function but for the cases of the Gaussian and sharp-k space filter.

Figure 7 shows the 2D EZL mass functions at $z=0$ for three different filter 


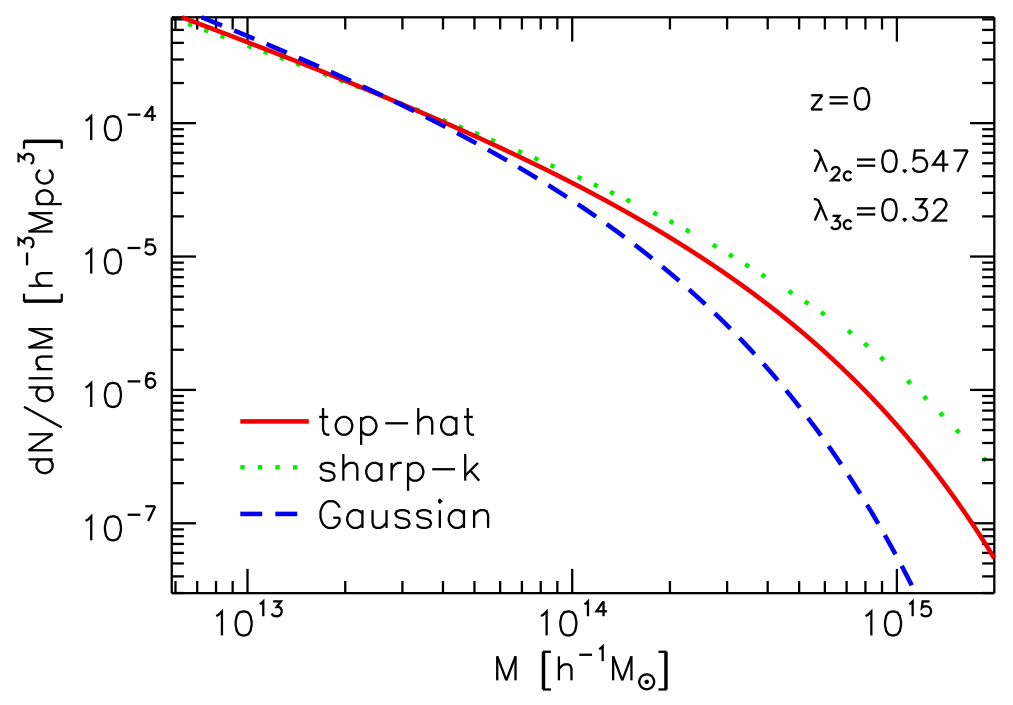

Figure 7. Variation of the 2D EZL mass function with the filter shape. The same values of the free parameters that was determined in section 4.1 by using the top-hat filter is applied to all three filter cases.

cases. For these plots, we use the same values of $\left\{\lambda_{2 c}, \lambda_{3 c}\right\}$ determined for the case of the top-hat filter in section 4.1. As can be seen, for the Gaussian filter case the number densities of the bound halos in the high-mass section $\left(M \geq 10^{14} h^{-1} M_{\odot}\right)$ are significantly reduced while those of the low-mass halos increase. In contrast, for the sharp- $k$ space filter case the trend becomes reversed: more high-mass halos and less low-mass halos than for the top-hat filter case.

To see if the success of the EZL formula is not limited to the top-hat filter case, we repeat the whole fitting process described in section 4.1 to determine the barrier heights of the 2D EZL formula but for the Gaussian and the sharp- $k$ space filter cases. Figures 8 and 9 show the 2D EZL mass functions (solid line) with the best-fit parameters at three different redshifts for the Gaussian and sharp- $k$ space filter cases, respectively. In each figure, the N-body results from the Millennium and the MICE simulations are also shown as dots (in the top and bottom panels, respectively). The best-fit parameters for both of the cases are different from those for the top-hat filter case. The higher value of $\lambda_{2 c}$ and lower value of $\lambda_{3 c}$ for the sharp- $k$ filter case, while the lower values of $\lambda_{2 c}$ and $\lambda_{3 c}$ for the Gaussian filter case. Anyway, even for the Gaussian and sharp- $k$ space filter case the 2D EZL mass functions with the constant values of the parameters at all redshifts are in excellent agreements with the N-body results. 


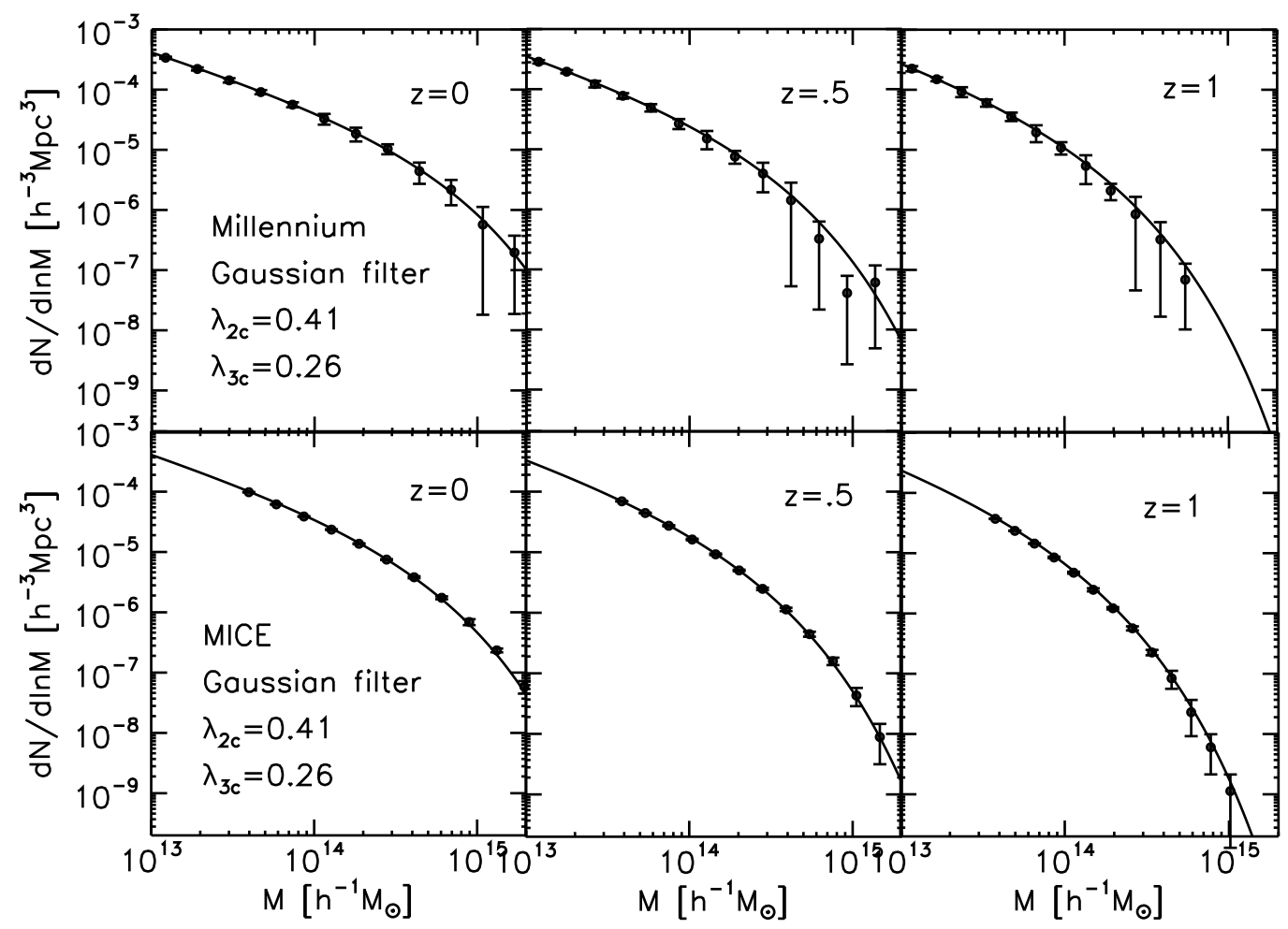

Figure 8. Same as the top panels of figures 5-6 but for the case of the Gaussian filter.

\section{Discussion}

Despite its excellent agreements with various N-body results, it is not a physical model but only a phenomenological fitting formula for the halo mass function since the bestfit values of its parameters are not related to the physics of real halo formation process. A critical reader might then ask what the advantage of using such a more complicated matrix equation as equation (3.1) for the evaluation of the halo mass function rather than using those simpler formulae suggested in the previous works. The merit of our EZL model is that it automatically satisfies the normalization constraint, as mentioned in 3, providing accurate fits with fewer fitting parameters than the previous models. Note that although the previous formulae of ref. [25] and ref. [27] have four fitting parameters including the normalization factor, their accuracy levels are not higher than our 2D EZL model which has only two parameters. Moreover, the empirically determined values of the free parameters of our EZL model have turned out to be independent of redshift and background cosmology. In our companion paper (S. Lim \& J. Lee 2012 in preparation), we have found that the same best-fit values of the parameters work even for a cosmology with primordial non-Gaussianity.

At any rate, to develop a physical model for the halo mass function within the 


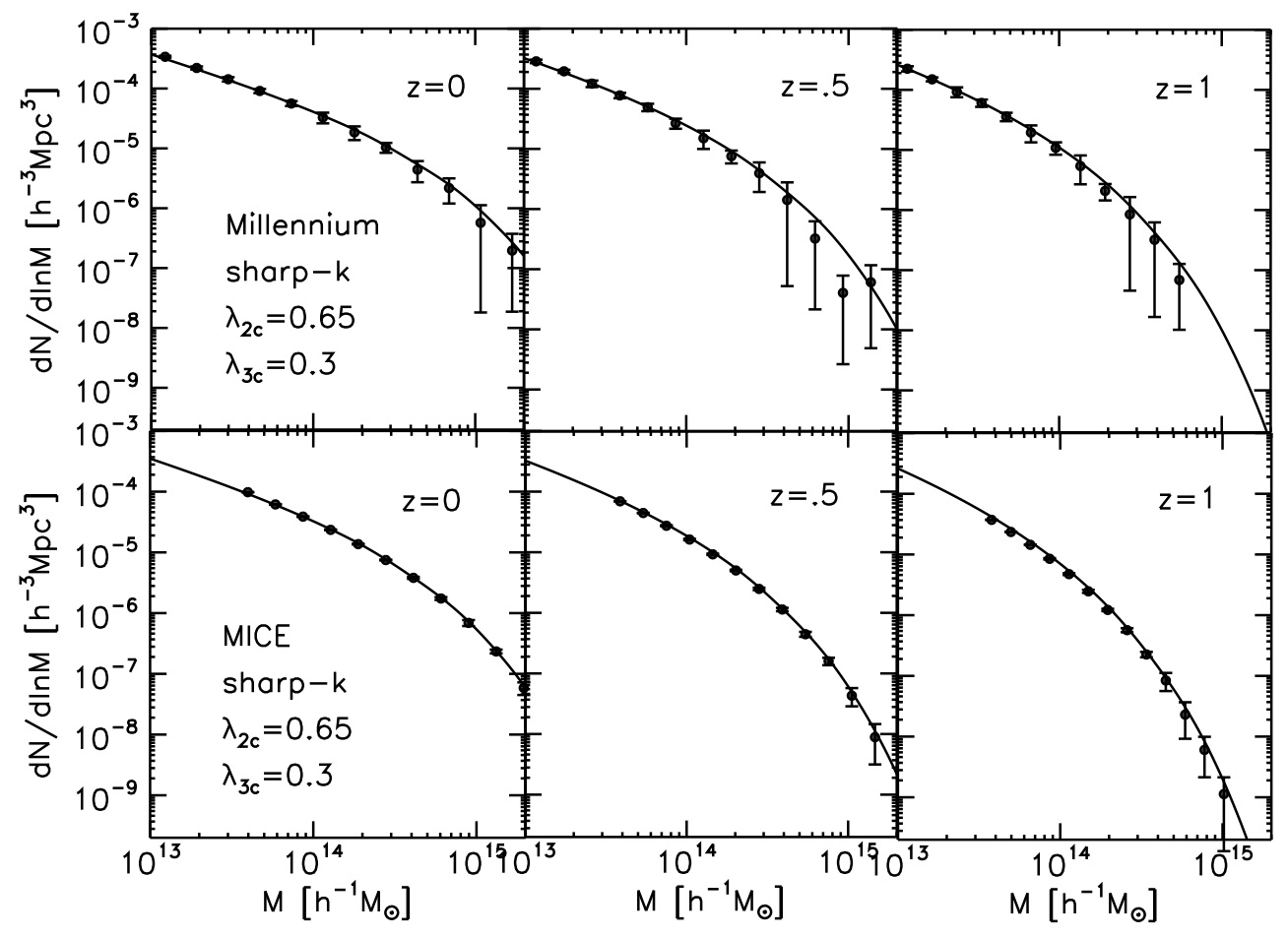

Figure 9. Same as figure 8 but for the case of the sharp- $k$ space filter.

framework of the Zel'dovich approximation, it will be evidently necessary to describe the growth of the initial shear eigenvalues by three dimensional non-Markovian random walks. It has been recently proved by ref. [17] that neglecting the correlations among random walks in the excursion set theory can significantly flaw analytic evaluation of the halo mass function. As an example, ref. [17] demonstrated that in the asymptotic limit of completely correlated random walks the excursion set theory in fact recovers the original PS mass function without the normalization factor of 2 . Regarding the normalization issue that the overall amplitude of the excursion set mass function for the non-Markovian case is substantially lower than the numerical results, ref. [19] has argued that it should imply the limitation of the excursion set approach itself: Due to the wrong assumption that the differential mass function $d N / d M$ on the right hand side of equation (2.1) can be simply related to the statistics of randomly placed cells on which the calculation of the differential volume fraction $f(\sigma)$ in the left-hand side of equation (2.1) is based, the excursion set mass function for the realistic non-Markovian case underestimates the abundance of bound halos.

The recent work of [18] may provide a clue to how to incorporate the nonMarkovian nature of random walks of shear eigenvalues into the Zel'dovich model. Ref. [18] have derived a very simple and accurate analytic model for the distribution of the non-Markovian random walks that first cross a given collapse barrier of arbitrary shape, and claimed that their model is valid even for the case that the collapse barrier is not a function of linear density contrast but of other initial quantities. Our future 
work is in the direction of finding a relation of the shear eigenvalues to the physics of true halo formation by extending the work of [18] to the case of three dimensional non-Markovian random walks of shear eigenvalues [see also 43].

\section{Acknowledgments}

We thank an anonymous referee who helped us improve significantly the original manuscript. We acknowledge the use of data from the Millennium and the MICE simulations that are publicly available at http://www.millennium.com,

and http://www.ice.cat/mice, respectively. The Millennium Simulation analyzed in this paper was carried out by the Virgo Supercomputing Consortium at the Computing Center of the Max-Planck Society in Garching, Germany. The computer code which evaluates the EZL halo mass functions will be provided upon request. This work was supported by the National Research Foundation of Korea (NRF) grant funded by the Korea government (MEST, No.2012-0004196) and partially by the research grant from the National Research Foundation of Korea to the Center for Galaxy Evolution Research (NO. 2010-0027910).

\section{References}

[1] P. J. E. Peebles, From Precision Cosmology to Accurate Cosmology, 2008 proceedings of the Moriond Conference on the Cosmological Models [astro-ph/0208037]

[2] Press W H and Schechter P, Formation of galaxies and clusters of galaxies by self-similar gravitational condensation, 1974 Astrophys. J. 187425

[3] Bond J R, Cole S, Efstathiou G and Kaiser N, Excursion set mass functions for hierarchical Gaussian fluctuations, 1991 Astrophys. J. 379440

[4] Peacock J A and Heavens A F, Alternatives to the Press-Schechter cosmological mass function, 1990 Mont. Not. Roy. Astron. 243133

[5] Jedamzik K, The Cloud-in-Cloud Problem in the Press-Schechter Formalism of Hierarchical Structure Formation, 1995 Astrophys. J. 4481

[6] Yano T, Nagashima M, and Gouda N, Limitations of the Press-Schechter Formalism, 1996 Astrophys. J. 4661

[7] Audit E, Teyssier R and Alimi J M, Non-linear dynamics and mass function of cosmic structures. I. Analytical results, 1997 Astron. E Astrophys. 325439

[8] Monaco P., A Lagrangian Dynamical Theory for the Mass Function of Cosmic Structures - I. Dynamics, 1997 Mont. Not. Roy. Astron. 287753

[9] Monaco P., A Lagrangian dynamical theory for the mass function of cosmic structures - II. Statistics, 1997 Mont. Not. Roy. Astron. 290439

[10] Chiueh T and Lee J, On the Nonspherical Nature of Halo Formation, 2001 Astrophys. J. 55583 
[11] Sheth R K, Mo H J and Tormen G, Ellipsoidal collapse and an improved model for the number and spatial distribution of dark matter haloes, 2001 Mont. Not. Roy. Astron. Soc. 323

[12] Maggiore M and Riotto A, The halo mass function from excursion set theory. I. Gaussian fluctuations with non-Markovian dependence on the smoothing scale, 2010 Astrophys. J. 711907

[13] Maggiore M and Riotto A, The Halo mass function from excursion set theory. II. The diffusing barrier 2010 Astrophys. J. 717515 [arXiv:0903.1250]

[14] Maggiore M and Riotto A, The halo mass function from excursion set theory. III. non-Gaussian fluctuations, 2010 Astrophys. J. 717526

[15] Corasaniti P S and Achitouv I, Toward a universal formulation of the halo mass function, 2011, Phys. Rev. Lett. 106241302

[16] Corasaniti P S and Achitouv I, Excursion set halo mass function and bias in a stochastic barrier model of ellipsoidal collapse, 2011 Phys. Rev. D 84023009

[17] Paranjape A, Lam T Y and Sheth R K Halo abundances and counts-in-cells: the excursion set approach with correlated steps, 2012 Mont. Not. Roy. Astron. Soc. 420 1429

[18] Musso M and Sheth R K, One step beyond: the excursion set approach with correlated steps, 2012 Mont. Not. Roy. Astron. Soc. 423 L102

[19] Paranjape A and Sheth R K Peaks theory and the excursion set approach, 2012 [arXiv:1206.3506]

[20] Lee J and Shandarin S F, The Cosmological Mass Function in the Zel'dovich Approximation, 1998 Astrophys. J. $\mathbf{5 0 0 ~} 4$

[21] Sheth R K and Tormen G, Large-scale bias and the peak background split, 1999 Mont. Not. Roy. Astron. 308119

[22] Reed D, Gardner J, Quinn T, Stadel J, Fardal M, Lake G, and Governato F, Evolution of the mass function of dark matter haloes, 2003 Mont. Not. Roy. Astron. Soc. 346565

[23] Jenkins A et al., The mass function of dark matter haloes, 2001 Mont. Not. Roy. Astron. Soc. 321372

[24] Warren M S, Abazajian K, Holz D E, and Teodoro L, Precision Determination of the Mass Function of Dark Matter Halos, 2006 Astrophys. J. 646881

[25] Tinker J L et al., Toward a halo mass function for precision cosmology: The limits of universality, 2008 Astrophys. J. 688709

[26] Crocce M, Fosalba P, Castander F J, and Gaztanaga E, Simulating the Universe with MICE: the abundance of massive clusters, 2010 Mont. Not. Roy. Astron. Soc. 4031353

[27] Pillepich A, Porciani C and Hahn O, Halo mass function and scale-dependent bias from N-body simulations with non-Gaussian initial conditions, 2010 Mont. Not. Roy. Astron. Soc. 402191

[28] Lee J, The relative abundance of isolated cluster as a probe of Dark energy, 2012 Astrophys. J. 75240 
[29] Zel'dovich Y B, Gravitational instability: An approximate theory for large density perturbations., 1970 Astron. \& Astrophys. 584

[30] Komatsu E et al., Seven-year Wilkinson microwave anisotropy probe (WMAP) observations: Cosmological interpretation, 2011 Astrophys. J. Supp. 19218

[31] Lewis A and Bridle S, Cosmological parameters from CMB and other data: A Monte Carlo approach, 2005 Phys. Rev. D. 66103511

[32] Eke V R, Cole S, and Frenk C S, Cluster evolution as a diagnostic for Omega, 1996 Mont. Not. Roy. Astron. Soc. 282263

[33] Davis M, Efstathiou G, Frenk C S, and White S D M., The evolution of large-scale structure in a universe dominated by cold dark matter, 1985 Astrophys. J. 292371

[34] Lacey C and Cole S, Merger Rates in Hierarchical Models of Galaxy Formation - Part Two - Comparison with N-Body Simulations, 1994 Mont. Not. Roy. Astron. 271676

[35] Dunkley J et al., Five-Year Wilkinson Microwave Anisotropy Probe Observations: Likelihoods and Parameters from the WMAP Data, 2009 Astrophys. J. 180306

[36] Bond J R and Myers S T, The Peak-Patch Picture of Cosmic Catalogs. I. Algorithms, 1996 Astrophys. J. 1031

[37] Porciani C, Dekel A, and Hoffman Y, Testing tidal-torque theory - II. Alignment of inertia and shear and the characteristics of protohaloes, 2002 Mont. Not. Roy. Astron. Soc. 332339

[38] Lim S and Lee J, The excursion set mass function of superclusters, [arXiv:1201.1382]

[39] Doroshkevich A G, Spatial structure of perturbations and origin of galactic rotation in fluctuation theory, 1970 Astrophyzika 3175

[40] Desjacques V, Environmental dependence in the ellipsoidal collapse model, 2008 Mont. Not. Roy. Astron. 388638

[41] Desjacques V and Smith R E, Statistical properties of the linear tidal shear, 2008 Phys. Rev. D. 66103511

[42] Springel V et al., Simulations of the formation, evolution and clustering of galaxies and quasars, 2005 Nature 435629

[43] Sheth R K, Chuen C K, and Scoccimarro R, Non-local Lagrangian bias, 2012 [arXiv:1207.7117] 